\title{
Semi-analytical modelling of piezoelectric excitation of guided waves
}

\author{
Michał K. Kalkowski ${ }^{* a}$ and Emiliano Rustighi ${ }^{a}$ and Timothy P. Waters ${ }^{a}$ \\ ${ }^{a}$ Institute of Sound and Vibration Research, University of Southampton, Highfield, \\ Southampton SO17 1BJ, United Kingdom
}

\begin{abstract}
Piezoelectric elements are a key component of modern non-destructive testing (NDT) and structural health monitoring (SHM) systems and play a significant role in many other areas involving dynamic interaction with the structure such as energy harvesting, active control, power ultrasonics or removal of surface accretions using structural waves. In this paper we present a wave-based technique for modelling waveguides equipped with piezoelectric actuators in which there is no need for common simplifications regarding their dynamic behaviour or mutual interaction with the structure. The proposed approach is based on the semi-analytical finite element (SAFE) method. We developed a new piezoelectric semi-analytical element and employed the analytical wave approach to model the distributed electric excitation and scattering of the waves at discontinuities. The model is successfully validated against an experiment on a beam-like waveguide with emulated anechoic terminations.
\end{abstract}

Keywords: piezoelectric excitation, guided wave propagation, semi-analytical finite element method, wave approach, smart structures

\section{INTRODUCTION}

The ability to describe the interaction between the piezo patch and the host structure accurately is an essential aspect of both the design and operational stages of piezo-equipped dynamic systems. Over the past decades this problem has been widely studied which resulted in a considerable number of modelling techniques. ${ }^{1}$ Among research published to date, two approaches can be distinguished. ${ }^{2}$ The first group considers the dynamics of the structure and piezo actuation separately, assuming that they are fully decoupled. A set of equivalent forces is applied to the substrate model by means of some coupling strategy. The second group of models accounts for the dynamic interaction between the actuator and the structure and is based on a solution to a boundary value problem.

Most of the models belonging to the first (decoupled) group refer to the quasi-static and pin-force assumptions presented first by Crawley and de Luis. ${ }^{3}$ The quasi-static assumption states that the actuator behaves quasistatically provided that it operates at frequencies far below its own resonances and is thin and light compared to the host structure. This also implies that the dynamics of the actuator are neglected and that for thin bonding layers the shear load transfer is confined at the ends of the actuator. The pin-force model was employed in the frequency domain spectral element method (SEM) to model coupled flexural-longitudinal-shear vibration of beams based on Timoshenko and Mindlin-Hermann theories. ${ }^{4-6}$

In recent decades the interest of researchers in the field was focused on modelling piezoelectric excitation at high frequencies, since piezoelectric elements have become a standard for high frequency wave-based NDT. ${ }^{7}$ To capture the structure dynamics, full elasticity equations need to be used. Pin-force assumption was applied to the integral transform solution of elasticity equations by Raghavan and Cesnik. ${ }^{8}$ Other researchers modelled Lamb wave excitation with a single PZT wafer as a boundary problem, incorporating full Lamb wave equations and accounting for the effect of the bonding layer. ${ }^{9-12}$ Dynamic contribution of the piezo actuator was included in the integral equation based methodology developed by Glushkov et al. ${ }^{13}$

Apart from analytical solutions, many researchers investigated piezo-excited waves using conventional FE. Being versatile and easily applicable to complex structures, this technique has important drawbacks in high

\footnotetext{
* e-mail: Michal.Kalkowski@soton.ac.uk
} 
frequency applications such as a very large computational cost and a limited insight into wave propagation phenomenon. Some of these can be circumvented in other numerical methods, e.g. the spectral finite element method. ${ }^{14,15}$ Various hybrid techniques employing full FE for modelling piezoelectric excitation and other techniques such as normal modes expansion or Local Interaction Simulation A pproach (LISA) for wave propagation have also been developed. ${ }^{16,17}$

The wide range of models briefly recalled above serves many purposes well in NDT/SHM applications, but has certain limitations both from the viewpoint of assumptions made and the model development (ability to model multi-component waveguides). Moreover, there are many applications such as removal of surface accretions using structural waves, where the actuators may operate close to their resonance frequencies and be of a similar to the host structure thickness which violates the quasi-static assumption.

In this paper we present a method to model structural wave excitation with piezo actuators which accounts for the dynamics of the actuator and its mutual interaction with the substrate (so far available only in FE) and at the same time takes advantage of the wave-based formulation. It also supports modelling multi-component structures without the need for employing full FE. The proposed coupled-field wave model originates from the piezoelectric constitutive equations, so a distributed voltage is applied directly. The approximations in place result from the finite element discretisation of the cross-section, the quasi-electrostatic approximation ${ }^{18}$ and the fact that the electric field in the propagation direction is neglected. To the best knowledge of the authors such a methodology has not been proposed to date.

\section{MODELLING STRUCTURAL DYNAMICS IN THE WAVE DOMAIN}

We first briefly describe the concept of formulating models in the wave domain. The advantage of this modelling paradigm becomes prominent at high frequencies, where many vibration modes exist or when the interest is in investigating wave propagation effects on their own. Analytical descriptions are available for a limited range of simple structures such as beams, rods or plates. Multi-component waveguides are therefore often modelled using conventional finite element method, which at high frequencies requires dense meshes and hence high computational resources. The alternative approach - wave-based modelling - exploits the fact that steadystate response of a structure is essentially a superposition of travelling waves. ${ }^{19,20}$ This enables reducing the computational load but also gaining an additional physical insight (wave propagation effects).

Building models in the wave domain involves two main stages. The first stage is the calculation of the wave characteristics - wavenumbers and wave mode shapes - of each uniform part of the waveguide (called a wave element in this paper) and the corresponding excited waves. The second stage is the assembly of the wave-based model performed using matrix algebra. The approach is illustrated in detail in Fig. 1, where a finite structure excited with a PZT actuator and a mechanical force is presented. The structure has a small notch and is fixed at its left end and attached via springs to a rigid wall at the other end.

The first step is to subdivide the structure into wave elements. The structure is split at any point at which a discontinuity (e.g. change of the cross-section or a mechanical force) occurs. Each wave element is then analysed using any capable method to obtain the wavenumbers and wave mode shapes (which together are called a wave basis in this paper). At this step, the excited wave amplitudes resulting from both piezoelectric and mechanical excitations are computed. Note that the excited waves are calculated as if the affected wave elements were infinite.

After the wave bases and the excited wave amplitudes are obtained, the reflection matrices are calculated based on the wave mode shapes and the boundary conditions. ${ }^{21}$ Scattering matrices describing wave conversion/reflection at the junctions are also found. ${ }^{21-23}$ To enable the calculation, the meshes of the overlapping parts of the subsequent adjacent cross-sections must conform to each other.

In the last step, the travelling waves amplitudes in each wave element are computed by means of matrix algebra. The term travelling waves denotes waves that represent superimposed contributions of subsequent reflections, scattering and appropriate excitations. The response within each of the elements is then computed from the wave mode shapes and the travelling wave amplitudes as denoted in Fig. 1.

In this paper we chose the semi-analytical finite element method for computing the wave bases and solving the excitation problem. The SAFE framework was proposed in the 1970s. ${ }^{24,25}$ The method is suitable for 


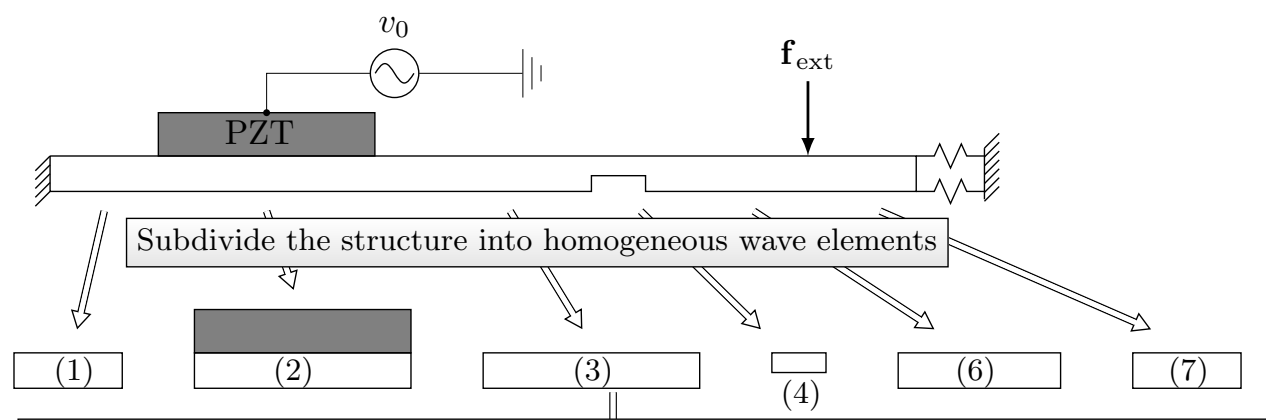

SAFE analysis of each wave element $\rightarrow$ wavenumbers, wave mode shapes, excited waves

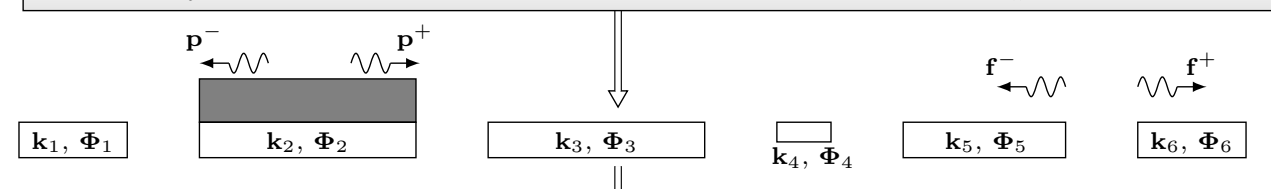

Calculation of the reflection matrices at the boundaries and scattering matrices at the joints
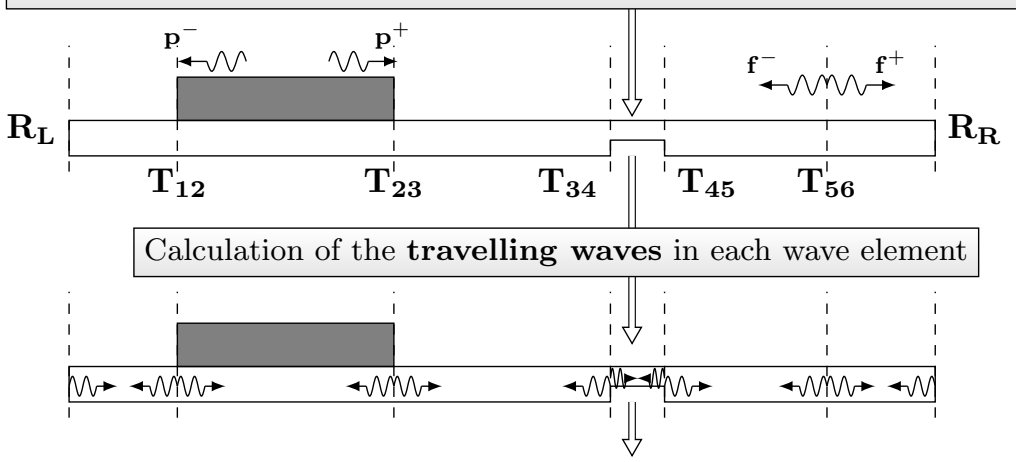

Calculation of the response in terms of wave mode shapes and travelling waves, e.g.:

$$
\mathbf{u}_{3}\left(z_{0}\right)=\boldsymbol{\Phi}_{3}^{+} \mathbf{a}_{3}^{+} \mathrm{e}^{-\jmath k_{3}^{+} z_{0}}+\boldsymbol{\Phi}_{3}^{-} \mathbf{a}_{3}^{-} \mathrm{e}^{-\jmath k_{3}^{+}\left(L-z_{0}\right)}
$$

Figure 1: The general concept of modelling structural dynamics in the wave domain

solving one-dimensional wave propagation problems for structures of an arbitrary cross-section, which is its great advantage over the analytical approaches. It utilises an FE-like procedure to discretise the plane crosssection and assumes a space-harmonic variation along the out-of-plane (waveguide) direction to find a steady-state solution of the equations of motion. The SAFE element has finite dimensions in the cross-section plane and is initially of an indefinite extent along the propagation direction. Further details of the method can be found in the literature. ${ }^{26-29}$

The SAFE method for elastic waveguides is well described and established within the scientific community. In this paper we formulate a SAFE element for materials with piezoelectric coupling to enable modelling piezoelectric excitation directly without a need for assumptions on the dynamics of the actuator or its mutual interaction with the structure. Wave based approach allows us to use both SAFE formulations (elastic and piezoelectric) to model complex multi-component smart structures equipped with piezoelectric actuators.

\section{SAFE FORMULATION FOR AN ELASTIC WAVEGUIDE WITH PIEZOELECTRIC COUPLING}

In the following paragraphs we derive the SAFE element for piezoelectric materials. Formulation for elastic waveguides may easily be recovered from the one given by setting all the piezoelectric material constants to zero. In most cases, the cross-sections of interest would contain both elastic and piezoelectric SAFE elements. The bonding conditions can be represented by an additional layer of either elastic/viscoelastic SAFE elements or 
spring SAFE elements. ${ }^{30}$ Global SAFE matrices are assembled in an FE-like manner, thus we focus our attention on presentation of the SAFE formulation for a single element only.

\subsection{Derivation of the SAFE element}

We assume that the cross-section of the structure lies in the $x-y$ plane and that the waves propagate along the $z$ direction (Fig. 2). The following derivation is written for the two-dimensional element, however other cases can be formulated by removing appropriate degrees of freedom and altering the element shape functions.

The displacement, strain and stress fields over a SAFE element are defined as: ${ }^{26}$

$$
\mathbf{u}=\left[\begin{array}{lll}
u_{x} & u_{y} & u_{z}
\end{array}\right]^{\top}, \quad \boldsymbol{\epsilon}=\left[\begin{array}{llllll}
\epsilon_{x x} & \epsilon_{y y} & \epsilon_{z z} & \gamma_{y z} & \gamma_{x z} & \gamma_{x y}
\end{array}\right]^{\top}, \quad \boldsymbol{\sigma}=\left[\begin{array}{llllll}
\sigma_{x x} & \sigma_{y y} & \sigma_{z z} & \sigma_{y z} & \sigma_{x z} & \sigma_{x y}
\end{array}\right]^{\top}
$$

where the time-harmonic term $\exp [\jmath \omega t]$ is omitted for brevity. Following the cross-section discretisation, the displacement at any point within the SAFE element is written as:

$$
\mathbf{u}(x, y, z)=\mathbf{N}(x, y) \mathbf{q}(z)
$$

where $\mathbf{N}(x, y)$ is the displacement shape function matrix (in the cross-section plane) and $\mathbf{q}(z)$ is the $z$-dependent nodal amplitudes vector. The strain is also expressed in terms of the shape functions and the nodal amplitudes:

$$
\boldsymbol{\epsilon}(x, y, z)=\left[\mathbf{L}_{x} \frac{\partial}{\partial x}+\mathbf{L}_{y} \frac{\partial}{\partial y}+\mathbf{L}_{z} \frac{\partial}{\partial z}\right] \mathbf{u}(x, y, z)=\mathbf{B}_{1} \mathbf{q}(z)+\mathbf{B}_{2} \frac{\partial}{\partial z} \mathbf{q}(z)
$$

where $\mathbf{L}_{x}, \mathbf{L}_{y}, \mathbf{L}_{z}$ indicate which displacement component is differentiated and can be deducted from Eq. (1). In a piezoelectric element each node has an additional degree of freedom $\varphi$ which represents the electrical potential. Analogously to Eq. (2), electrical potential in the waveguide $\boldsymbol{\vartheta}$ is expressed as

$$
\vartheta(x, y, z)=\mathbf{N}_{\varphi}(x, y) \varphi(z)
$$

The behaviour of a piezoelectric material is governed by the following constitutive equations: ${ }^{31}$

$$
\begin{aligned}
\boldsymbol{\sigma} & =\mathbf{C}^{\mathrm{E}} \boldsymbol{\epsilon}-\mathbf{e}^{\top} \mathbf{E} \\
\mathbf{D} & =\mathbf{e} \boldsymbol{\epsilon}+\boldsymbol{\varepsilon}^{\epsilon} \mathbf{E}
\end{aligned}
$$

where $\mathbf{C}^{\mathrm{E}}$, e, $\mathbf{E}, \mathbf{D}, \varepsilon^{\epsilon}$ are the stiffness matrix measured under zero-electric field (short-circuit) condition, piezoelectric stress coefficient matrix, electric field vector, electric displacement vector and permittivity matrix measured at zero-strain (clamped) condition, respectively.

The electric field is assumed to be quasi-static (curl $\mathbf{E}$ is zero) and thus it is expressed as a scalar gradient of the potential ${ }^{32}$

$$
\mathbf{E}(x, y, z)=-\nabla \boldsymbol{\vartheta}
$$

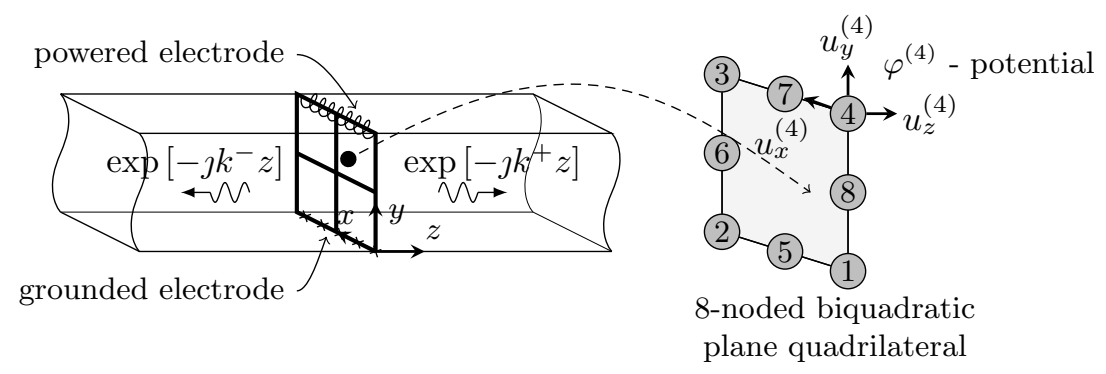

Figure 2: Waveguide with piezoelectric coupling modelled with SAFE - the coordinates system, labelling convention and degrees of freedom. 
Given the discretisation of the cross-section one writes ${ }^{33-35}$

$$
\mathbf{E}(x, y, z)=-\nabla \boldsymbol{\vartheta}=-\left[\begin{array}{lll}
\frac{\partial}{\partial x} & \frac{\partial}{\partial y} & \frac{\partial}{\partial z}
\end{array}\right]^{\top} \mathbf{N}_{\varphi} \boldsymbol{\varphi}
$$

where the electrical potential shape functions $\mathbf{N}_{\varphi}$ are chosen to be the same as the displacement shape functions N. In this paper the piezoelectric SAFE element is developed for modelling actuators made of bulk piezoelectric ceramic with two electroded surfaces. Driving voltage is applied across the thickness of the PZT element (the $y$ direction). To enable condensation of the potentials (though it is not necessary), the electric field is assumed not to change along the propagation direction $z$. By doing so, the contribution of the piezoelectric coefficient associated with the voltage gradient along $z$ is neglected, which is not expected to invoke a significant error for the calculation of excited waves. Eq. (7) is rewritten as

$$
\mathbf{E}(x, y, z)=-\left[\begin{array}{lll}
\mathbf{N}_{\varphi, x} & \mathbf{N}_{\varphi, y} & 0
\end{array}\right]^{\top} \boldsymbol{\varphi}=-\mathbf{B}_{\varphi} \boldsymbol{\varphi}
$$

Equations of motion are found with the aid of the virtual work and virtual electric potentials principles. ${ }^{32}$ Given the constitutive equations and appropriate natural and essential boundary conditions, ${ }^{23}$ the virtual work principle states that for every time $t$

$$
\overbrace{\int_{\Gamma_{\sigma}} \delta \mathbf{u}^{\top} \mathbf{t} \mathrm{d} \Gamma_{\sigma}}^{\substack{\text { work done by } \\
\text { external surface } \\
\text { tractions }}}+\overbrace{\delta \mathbf{q}^{\top} \mathbf{f}_{i}}^{\begin{array}{c}
\text { work done by } \\
\text { nodal forces }
\end{array}}=\overbrace{\int_{\mathrm{V}} \delta \mathbf{u}^{\top}(\rho \ddot{\mathbf{u}}) \mathrm{d} V}^{\begin{array}{c}
\text { work done by } \\
\text { inertia body forces }
\end{array}}+\overbrace{\int_{\mathrm{V}} \delta \boldsymbol{\epsilon}^{\top} \boldsymbol{\sigma} \mathrm{d} V}^{\begin{array}{c}
\text { work done by } \\
\text { the stress field }
\end{array}}
$$

where $\mathbf{t}$ is the external tractions vector, $\mathbf{f}_{i}$ is the vector of nodal forces applied at the ends of the considered section of the waveguide of length $L$ and $\rho$ is the density of the material.

Similarly, under the essential boundary conditions, ${ }^{23}$ the virtual electric potentials principle states that for every time $t$

$$
-\overbrace{\int_{\Gamma_{\varrho}} \delta \varphi^{\top} \varrho \mathrm{d} \Gamma_{\varrho}}^{\begin{array}{c}
\text { work done by } \\
\text { external surface } \\
\text { charges }
\end{array}}=\overbrace{\int_{\mathrm{V}} \delta \mathbf{E}^{\top} \mathbf{D} \mathrm{d} V}^{\begin{array}{c}
\text { work done by } \\
\text { the electric } \\
\text { displacement field }
\end{array}}
$$

where $\varrho$ is the external surface charge vector.

Appropriate terms are now substituted into Eqs. (9) and (10) with the expressions coming from the finite element discretisation of the cross-section. To clarify the formulation, the following notation is adopted which results from the integration of the matrices over the cross-sectional element domain $\Omega$ or appropriate element boundaries in case of mechanical or electrical surface loads $\left(\Gamma_{\sigma} \text { or } \Gamma_{\varrho} \text {, respectively }\right)^{23}$

$$
\begin{aligned}
& \mathbf{M}=\int_{\Omega} \mathbf{N}^{\top} \rho \mathbf{N} \mathrm{d} \Omega \quad \mathbf{K}_{f}=\int_{\Omega} \mathbf{B}_{2}^{\top} \mathbf{C B}_{1} \mathrm{~d} \Omega \quad \mathbf{K}_{0}=\int_{\Omega} \mathbf{B}_{1}^{\top} \mathbf{C B}_{1} \mathrm{~d} \Omega \\
& \mathbf{K}_{1}=\int_{\Omega} \mathbf{K}_{f}^{\top}-\mathbf{K}_{f} \mathrm{~d} \Omega \quad \mathbf{K}_{2}=\int_{\Omega} \mathbf{B}_{2}^{\top} \mathbf{C B}_{2} \mathrm{~d} \Omega \quad \mathbf{p}=\int_{\Gamma_{\sigma}} \mathbf{N}^{\top} \mathbf{N t}_{i} \mathrm{~d} \Gamma_{\sigma} \\
& \mathbf{K}_{0}^{u \varphi}=\mathbf{K}_{0}^{\varphi u \top}=\int_{\Omega} \mathbf{B}_{1}^{\top} \mathbf{e}^{\top} \mathbf{B}_{\varphi} \mathrm{d} \Omega \quad \mathbf{K}_{1}^{u \varphi}=\mathbf{K}_{1}^{\varphi u \top}=\int_{\Omega} \mathbf{B}_{2}^{\top} \mathbf{e}^{\top} \mathbf{B}_{\varphi} \mathrm{d} \Omega \quad \mathbf{K}^{\varphi \varphi}=\int_{\Omega} \mathbf{B}_{\varphi}^{\top} \varepsilon^{\mathrm{s}} \mathbf{B}_{\varphi} \mathrm{d} \Omega \\
& \Upsilon=\int_{\Gamma_{\varrho}} \varrho \mathrm{d} \Gamma_{\varrho}
\end{aligned}
$$


After some mathematical manipulations of Eq. (9) and Eq. (10) one can conclude the governing equation for wave propagation:

$$
\begin{aligned}
\mathbf{M} \frac{\partial^{2}}{\partial t^{2}} \mathbf{q}+\mathbf{K}_{0} \mathbf{q}+\mathbf{K}_{1} \frac{\partial}{\partial z} \mathbf{q}-\mathbf{K}_{2} \frac{\partial^{2}}{\partial z^{2}} \mathbf{q}+\mathbf{K}_{0}^{u \varphi} \boldsymbol{\varphi}-\mathbf{K}_{1}^{u \varphi} \frac{\partial}{\partial z} \boldsymbol{\varphi} & =\mathbf{p} \\
\mathbf{K}_{0}^{\varphi u} \mathbf{q}+\mathbf{K}_{1}^{\varphi u} \frac{\partial}{\partial z} \mathbf{q}-\mathbf{K}^{\varphi \varphi} \boldsymbol{\varphi} & =\mathbf{\Upsilon}
\end{aligned}
$$

and the natural boundary condition stating that at the ends of the considered section of the waveguide:

$$
\mathbf{q}=0 \quad \text { or } \quad \mathbf{f}_{i}=\mathbf{K}_{f} \mathbf{q}+\mathbf{K}_{2} \frac{\partial}{\partial z} \mathbf{q}+\mathbf{K}_{1}^{u \varphi} \boldsymbol{\varphi}
$$

which directly provides the expression for the resultant nodal forces.

Solution to Eq. (12) is performed using the Spatial Fourier Transform (SFT) defined as

$$
\mathcal{F}\{\mathbf{q}\}=\overline{\mathbf{q}}(k)=\int_{-\infty}^{\infty} \mathbf{q}(z) \exp [\jmath k z] \mathrm{d} z \quad \text { and } \quad \mathbf{q}=\frac{1}{2 \pi} \int_{-\infty}^{\infty} \overline{\mathbf{q}}(k) \exp [-\jmath k z] \mathrm{d} k
$$

Applying Eq. (14) to Eq. (12) and Eq. (13) and performing the time derivation under the harmonic motion assumption, one writes

$$
\left(\left[\begin{array}{cc}
-\omega^{2} \mathbf{M} & \mathbf{0} \\
\mathbf{0} & \mathbf{0}
\end{array}\right]+\left[\begin{array}{cc}
\mathbf{K}_{0}-\jmath k \mathbf{K}_{1}-(-\jmath k)^{2} \mathbf{K}_{2} & \mathbf{K}_{0}^{u \varphi}+\jmath k \mathbf{K}_{1}^{u \varphi} \\
\mathbf{K}_{0}^{\varphi u}-\jmath k \mathbf{K}_{1}^{\varphi u} & -\mathbf{K}^{\varphi \varphi}
\end{array}\right]\right)\left\{\begin{array}{l}
\overline{\mathbf{q}} \\
\overline{\boldsymbol{\varphi}}
\end{array}\right\}=\left\{\begin{array}{l}
\overline{\mathbf{p}} \\
\overline{\mathbf{\Upsilon}}
\end{array}\right\}
$$

and

$$
\overline{\mathbf{f}}_{i}=\mathbf{K}_{f} \overline{\mathbf{q}}-\jmath k \mathbf{K}_{2} \overline{\mathbf{q}}+\mathbf{K}_{1}^{u \varphi} \bar{\varphi}
$$

Eq. (15) is the governing equation for wave propagation in a piezoelectric waveguide in the wavenumber-frequency domain whereas the resultant nodal forces in the wavenumber-frequency domain can be calculated from Eq. (16).

The focus of the procedure is the application to piezoelectric actuators where the outer surfaces perpendicular to the propagation direction are electroded. It is then customary to express the above equation in terms of driving voltage which can be done by condensing out the potentials at the internal nodes. Static condensation procedure is standard and the details may readily be found in the literature. ${ }^{33,34,36}$ The tilde $\{\hat{\}}$ symbol in the following equations indicates that the matrix is amended to account for the condensed potentials. Note, however, that although condensation simplifies the notation and the solution procedure, it also involves the loss of sparsity of SAFE matrices. Therefore, from a numerical performance viewpoint condensation is not the optimal choice.

\subsection{Free waves and the wave basis}

Free waves in a waveguide with piezoelectric coupling in the short-circuit condition may be found by setting the voltage in the powered electrode to zero $(v=0)$ and assuming no external mechanical traction. This results in a quadratic eigenvalue problem (QEP)

$$
\left[\tilde{\mathbf{K}}_{0}+\lambda \tilde{\mathbf{K}}_{1}-\lambda^{2} \tilde{\mathbf{K}}_{2}-\omega^{2} \mathbf{M}\right] \boldsymbol{\phi}=\mathbf{0}
$$

where $\phi$ is the eigenvector and $\lambda=-\jmath k$.

QEP from Eq. (17) is most commonly solved by companion form linearisation. ${ }^{37}$ A new eigenvector is introduced as $\hat{\phi}=\left[\begin{array}{ll}\phi & \lambda \phi\end{array}\right]^{\top}$ and the SAFE governing equation is rewritten as a generalised eigenvalue problem (GEP)

$$
\left(\left[\begin{array}{cc}
\mathbf{0} & \tilde{\mathbf{K}}_{0}-\omega^{2} \mathbf{M} \\
\tilde{\mathbf{K}}_{0}-\omega^{2} \mathbf{M} & \tilde{\mathbf{K}}_{1}
\end{array}\right]-\lambda\left[\begin{array}{cc}
\tilde{\mathbf{K}}_{0}-\omega^{2} \mathbf{M} & \mathbf{0} \\
\mathbf{0} & \tilde{\mathbf{K}}_{2}
\end{array}\right]\right)\left\{\begin{array}{c}
\phi \\
\lambda \phi
\end{array}\right\}=\mathbf{0}
$$

or more concisely as

$$
[\mathbf{A}(\omega)-\lambda \mathbf{B}(\omega)] \hat{\boldsymbol{\phi}}=\mathbf{0}
$$


where $\mathbf{A}, \mathbf{B}$ are of dimensions $2 n \times 2 n$ and $n$ is the number of the displacement degrees of freedom in the cross-section.

The GEP from Eq. (19) can be solved using any of the scientific computing packages. For a given frequency $\omega$ one finds a set of $2 n$ eigenvalues $\lambda_{i}$ (associated with $n$ positive- and $n$ negative-going waves), and $2 n \times 2 n$ matrices of the corresponding right and left eigenvectors denoted by $\hat{\boldsymbol{\Phi}}$ and $\hat{\boldsymbol{\Psi}}$, respectively. The right eigenvectors $\hat{\phi}_{i}$ are columns of $\hat{\boldsymbol{\Phi}}$ and left eigenvectors $\hat{\boldsymbol{\psi}}_{i}$ are rows of $\hat{\boldsymbol{\Psi}}$. A more detailed description of the properties of the matrices and free wave solutions can be found in the literature. ${ }^{23}$

\subsection{Excitation of structural waves in an elastic waveguide covered with a piezoelectric layer powered over a finite length}

We consider a steady-state response in an infinite elastic layer covered over its whole length by an electroded piezoelectric layer (Fig. 3). A finite section of the electrode is driven with a time-harmonic voltage $v_{0}$. Note that the 'driving' section is electrically isolated from adjacent parts of the waveguide which are in the short-circuit condition. Vectors $\mathbf{p}^{-}$and $\mathbf{p}^{+}$represent waves generated by the distributed patch-like piezoelectric excitation as they enter the non-powered region. A local coordinate system $\left(z^{\prime}\right)$ is introduced with the origin in the middle of the length of the piezoelectric actuator. The spatial distribution of voltage $v\left(z^{\prime}\right)$ is a rectangular box function as shown in Fig. 3, such that

$$
v\left(z^{\prime}\right)= \begin{cases}v_{0} & \text { if } z^{\prime} \leq|L / 2| \\ 0 & \text { otherwise }\end{cases}
$$

Excited wave amplitudes are found by solving the SAFE equation in the wavenumber-frequency domain directly, given the spatial distribution of voltage (the solution is similar to the distributed force case ${ }^{38}$ ). Let us first recall the top row of the governing equation for wave propagation in the piezoelectric waveguide in Eq. (15), assuming no external tractions for the sake of clarity of the formulation

$$
\left(\tilde{\mathbf{K}}_{0}-\jmath k \tilde{\mathbf{K}}_{1}-(-\jmath k)^{2} \tilde{\mathbf{K}}_{2}-\omega^{2} \mathbf{M}\right) \overline{\mathbf{q}}=\left(-\tilde{\mathbf{K}}_{0}^{u \varphi}-\jmath k \tilde{\mathbf{K}}_{1}^{u \varphi}\right) \bar{v}
$$

where $\bar{v}$ is the SFT of the voltage spatial distribution given by

$$
\bar{v}=\int_{-\infty}^{\infty} v\left(z^{\prime}\right) \exp \left[\jmath k z^{\prime}\right] \mathrm{d} z^{\prime}=2 v_{0} \frac{\sin (k L / 2)}{k}
$$

The solution is performed on the linearised form of Eq. (21) which enables the use of GEP properties

$$
[\mathbf{A}(\omega)-(-\jmath k) \mathbf{B}(\omega)] \overline{\mathbf{q}}=\left[\begin{array}{c}
0 \\
-\tilde{\mathbf{K}}_{0}^{u \varphi}-\jmath k \tilde{\mathbf{K}}_{1}^{u \varphi}
\end{array}\right] \bar{v}
$$

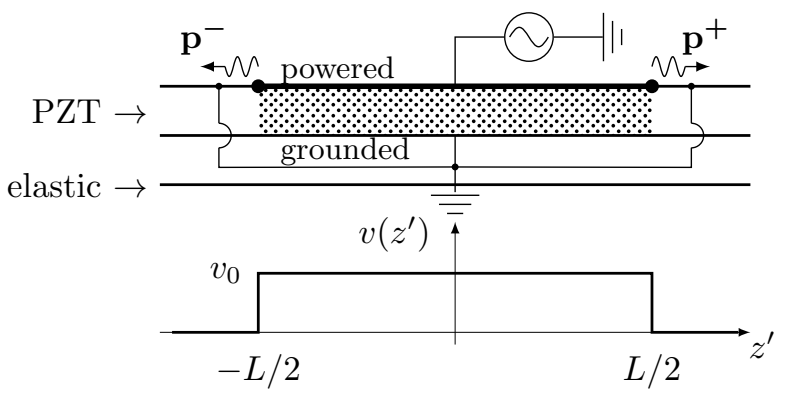

Figure 3: Elastic waveguide covered with a piezoelectric layer driven over a finite length. Excited waves amplitudes and voltage spatial distribution. 
After pre-multiplying by $\hat{\boldsymbol{\psi}}_{\mathbf{i}}$ and exploiting the orthogonality of the eigenvectors with respect to $\mathbf{B}$ one writes the solution as

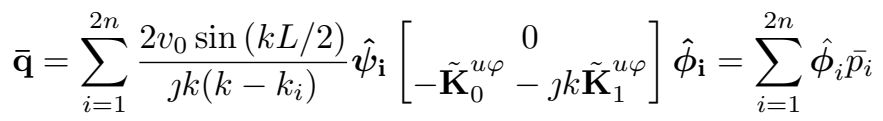

where $\bar{v}$ was substituted with the expression from Eq. (22).

The form of Eq. (24) is similar in appearance to the wave basis expressions, i.e. the solution to the voltage excited wave problem is already written as a weighted superposition of the travelling waves. The modal weighting factor representing the amplitude of wave $i$ in the space domain is found via the inverse SFT defined in Eq. (14)

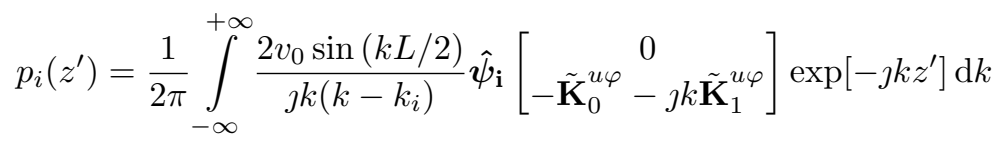

The above integral is evaluated using Cauchy's residue theorem ${ }^{39}$ yielding excited wave amplitudes in the space domain

$$
\begin{aligned}
& p_{i}^{+}\left(z^{\prime}\right)=-\frac{2 v_{0} \sin \left(k_{i}^{+} L / 2\right)}{k_{i}^{+}} \hat{\boldsymbol{\psi}}_{\mathbf{i}}^{+}\left[\begin{array}{c}
0 \\
-\tilde{\mathbf{K}}_{0}^{u \varphi}-\jmath k_{i}^{+} \tilde{\mathbf{K}}_{1}^{u \varphi}
\end{array}\right] \exp \left[-\jmath k_{i}^{+} z^{\prime}\right] \\
& p_{i}^{-}\left(z^{\prime}\right)=\frac{2 v_{0} \sin \left(k_{i}^{-} L / 2\right)}{k_{i}^{-}} \hat{\boldsymbol{\psi}}_{\mathbf{i}}^{-}\left[\begin{array}{c}
0 \\
-\tilde{\mathbf{K}}_{0}^{u \varphi}-\jmath k_{i}^{-} \tilde{\mathbf{K}}_{1}^{u \varphi}
\end{array}\right] \exp \left[-\jmath k_{i}^{-} z^{\prime}\right]
\end{aligned}
$$

Excited wave amplitudes $p_{i}^{+}\left(z^{\prime}\right), p_{i}^{-}\left(z^{\prime}\right)$ are expressed with reference to the local coordinate system as depicted in Fig. 3. In order to obtain the excited waves amplitudes as they appear at the ends of the electrically driven region, they need to be multiplied by appropriate space harmonic term representing propagation over $L / 2$

$$
\begin{aligned}
& p_{i}^{+}=-\frac{v_{0}\left(1-\exp \left[-\jmath k_{i}^{+} L\right]\right)}{\jmath k_{i}^{+}} \hat{\boldsymbol{\psi}}_{\mathbf{i}}^{+}\left[\begin{array}{c}
0 \\
-\tilde{\mathbf{K}}_{0}^{u \varphi}-\jmath k_{i}^{+} \tilde{\mathbf{K}}_{1}^{u \varphi}
\end{array}\right] \\
& p_{i}^{-}=\frac{v_{0}\left(\exp \left[\jmath k_{i}^{-} L\right]-1\right)}{\jmath k_{i}^{-}} \hat{\boldsymbol{\psi}}_{\mathbf{i}}^{-}\left[\begin{array}{c}
0 \\
-\tilde{\mathbf{K}}_{0}^{u \varphi}-\jmath k_{i}^{-} \tilde{\mathbf{K}}_{1}^{u \varphi}
\end{array}\right]
\end{aligned}
$$

Eq. (27) provides expressions for wave amplitudes excited by voltage uniformly distributed over $L$ in an infinite piezo-covered waveguide as they appear at the ends of the powered region. Identical expressions can be found from convolving the response to a point voltage excitation with the spatial voltage distribution.

\section{EXCITATION OF STRUCTURAL WAVES WITH A FINITE LENGTH ACTUATOR - AN ILLUSTRATIVE EXAMPLE}

In this section we present how the SAFE element developed in the previous section is used to calculate piezoelectric excitation in waveguides. The approach is demonstrated on an infinite waveguide with a rectangular cross-section equipped with a finite piezo actuator. The structure consists of three wave elements, i.e. two (identical) elastic elements and one elastic element covered with a piezoelectric layer. Each wave element is analysed using SAFE to find the wave bases and the amplitudes of waves generated by the voltage excitation as outlined in the previous section.

In the following paragraphs we present the calculation of the travelling waves and desired responses in the considered structure. There are two possible outputs of such calculation, namely either the excited wave field propagating in the elastic substrate or the electrical impedance of the actuator. Both cases are considered below. 


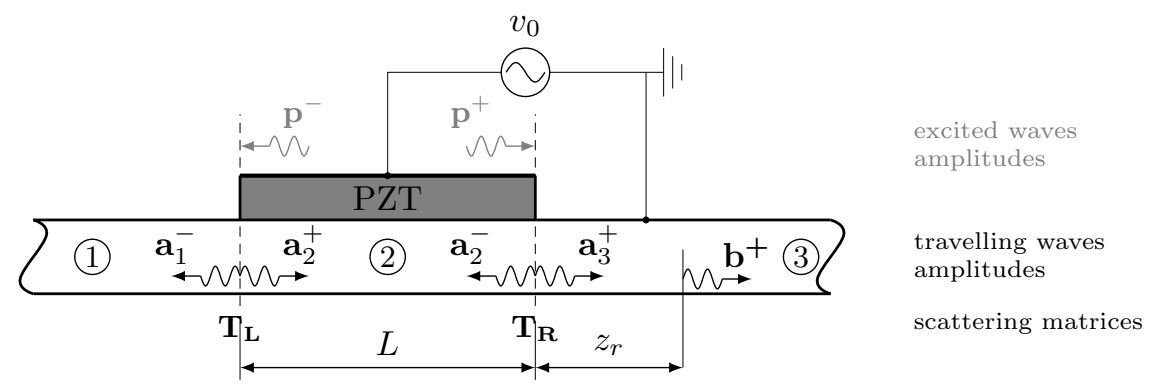

Figure 4: Infinite waveguide excited with a piezoelectric actuator - schematic diagrams for calculating the travelling waves outside the PZT-covered section; the excited waves $\mathbf{p}^{+}, \mathbf{p}^{-}$are defined at the edges of the actuator just before they are incident on the discontinuity.

\subsection{The response outside the piezo-covered region}

The interest is in finding the wave amplitudes induced in the substrate waveguide at a position $z_{r}$ to the right of the actuator, denoted hereafter by $\mathbf{b}^{+}$. Travelling waves at different stages and excited waves at the ends of the actuator are depicted in Fig. 4.

The wave amplitudes resulting from the distributed voltage excitation $\mathbf{p}^{-}, \mathbf{p}^{+}$as found in Section 3.3 are immediately incident upon the discontinuities at the ends of the piezoelectric actuator, and therefore scattered according to the appropriate scattering matrices. ${ }^{21,22}$

The boundary conditions are ideally absorbing (located at $\pm \infty$ ), hence there is no negative-going wave in element 3 and the response of the structure at $z_{r}$ is written in the wave domain as

$$
\mathbf{q}\left(z_{r}\right)=\boldsymbol{\phi}^{+} \mathbf{b}^{+}\left(z_{r}\right)=\boldsymbol{\phi}^{+} \boldsymbol{\tau}\left(z_{r}\right) \mathbf{a}_{3}^{+}
$$

where $\boldsymbol{\tau}\left(z_{r}\right)=\operatorname{diag}\left(\exp \left[-\jmath k_{i} z_{r}\right]\right)$.

To obtain the response at a desired location $z_{r}$, one needs to find the travelling wave amplitude $\mathbf{a}_{\mathbf{3}}{ }^{+}$first. We write it in terms of the excited wave and the positive travelling wave in element 2 :

$$
\mathbf{a}_{3}^{+}=\mathbf{T}_{\mathrm{R}}^{12} \mathbf{p}^{+}+\mathbf{T}_{\mathrm{R}}^{12} \boldsymbol{\tau}(L) \mathbf{a}_{2}^{+}
$$

where

$$
\mathbf{a}_{2}^{+}=\mathbf{R}_{\mathrm{L}}^{22} \mathbf{p}^{-}+\mathbf{R}_{\mathrm{L}}^{22} \boldsymbol{\tau}(L) \mathbf{a}_{2}^{-}
$$

and

$$
\mathbf{a}_{2}^{-}=\mathbf{R}_{\mathrm{R}}^{11} \mathbf{p}^{+}+\mathbf{R}_{\mathrm{R}}^{11} \boldsymbol{\tau}(L) \mathbf{a}_{2}^{+}
$$

After appropriate manipulations we obtain

$$
\mathbf{a}_{3}^{+}=\mathbf{T}_{\mathrm{R}}^{12} \mathbf{p}^{+}+\mathbf{T}_{\mathrm{R}}^{12} \boldsymbol{\tau}(L)\left[\mathbf{I}-\mathbf{R}_{\mathrm{L}}^{22} \boldsymbol{\tau}(L) \mathbf{R}_{\mathrm{R}}^{11} \boldsymbol{\tau}(L)\right]^{-1}\left[\mathbf{R}_{\mathrm{L}}^{22} \mathbf{p}^{-}+\mathbf{R}_{\mathrm{L}}^{22} \boldsymbol{\tau}(L) \mathbf{R}_{\mathrm{R}}^{11} \mathbf{p}^{+}\right]
$$

which after substituting into Eq. (28) leads to the desired response.

\subsection{The response within the piezo-covered region: electrical impedance extraction}

Many engineering applications take advantage of the electromechanical coupling in piezoelectric elements to sense the dynamic behaviour of the structure by means of the frequency dependent electrical impedance of the PZT actuator. We show how the presented model can be used to extract the electrical impedance.

The driving voltage $v$ is kept fixed, whereas the current drawn is obtained from the second row of Eq. (15) which provides an expression for the total reaction charge on the electrode. Since the reaction charge needs to be summed over the length of the actuator, a methodology for evaluating the response inside the actuator is required. At each location $z_{i}$ along the length of the PZT-covered wave element we split it into two parts: the 


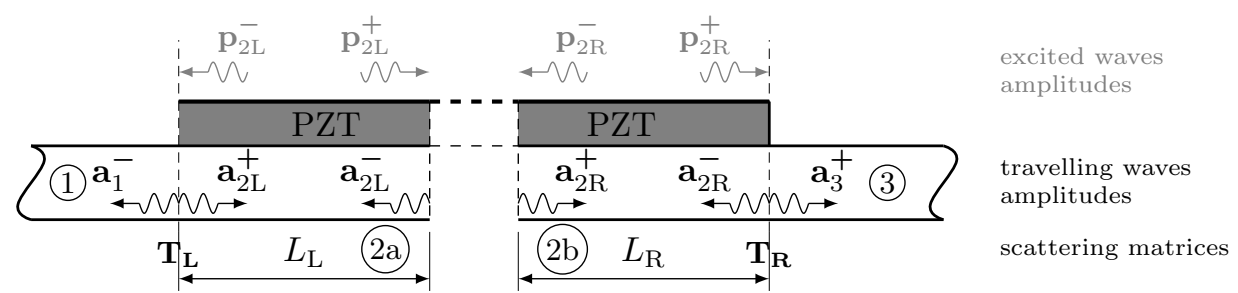

Figure 5: Infinite waveguide excited with a piezoelectric actuator - schematic diagrams for calculating the travelling waves inside the PZT-covered section; the excited waves $\mathbf{p}_{2 \mathrm{~L}}^{+}, \mathbf{p}_{2 \mathrm{~L}}^{-}, \mathbf{p}_{2 \mathrm{R}}^{+}, \mathbf{p}_{2 \mathrm{R}}^{-}$are defined at the edges of the actuator just before they are incident on the discontinuity.

one to the left from $z_{i}\left(z \leq z_{i}\right)$ and that to the right from $z_{i}\left(z \geq z_{i}\right){ }^{38}$ Excited waves are calculated separately for each part using the framework presented in the previous paragraphs and substituting the correct lengths depending on $z_{i}$. A schematic diagram for the calculation is shown in Fig. 5.

The excited waves $\mathbf{p}_{2 \mathrm{~L}}^{+}, \mathbf{p}_{2 \mathrm{~L}}^{-}, \mathbf{p}_{2 \mathrm{R}}^{+}, \mathbf{p}_{2 \mathrm{R}}^{-}$as depicted in Fig. 5 are the wave amplitudes excited in an infinite waveguide covered by the piezoelectric layer powered over a finite length (Section 3.3) and in case of a finite piezoelectric actuator they are immediately incident upon a discontinuity.

We start the calculation from writing the expressions for travelling waves at different stages as

$$
\begin{array}{ll}
\mathbf{a}_{2 R}^{+}=\mathbf{p}_{2 L}^{+}+\boldsymbol{\tau}\left(L_{L}\right) \mathbf{a}_{2 L}^{+} & \mathbf{a}_{2 R}^{-}=\mathbf{R}_{R}^{11} \mathbf{p}_{2 R}^{+}+\mathbf{R}_{R}^{11} \boldsymbol{\tau}\left(L_{R}\right) \mathbf{a}_{2 R}^{+} \\
\mathbf{a}_{2 L}^{-}=\mathbf{p}_{2 R}^{-}+\boldsymbol{\tau}\left(L_{R}\right) \mathbf{a}_{2 R}^{-} & \mathbf{a}_{2 L}^{+}=\mathbf{R}_{L}^{22} \mathbf{p}_{2 L}^{-}+\mathbf{R}_{L}^{22} \boldsymbol{\tau}\left(L_{L}\right) \mathbf{a}_{2 L}^{-}
\end{array}
$$

After some algebraic manipulations of the above equations we obtain the expressions for the positive- and negative-going waves at a location $z_{i}$ within the piezoelectric actuator

$$
\begin{aligned}
& \mathbf{a}_{2 L}^{-}=\left[\mathbf{I}-\boldsymbol{\tau}\left(L_{R}\right) \mathbf{R}_{R}^{11} \boldsymbol{\tau}(L) \mathbf{R}_{L}^{22} \boldsymbol{\tau}\left(L_{L}\right)\right]^{-1}\left\{\mathbf{p}_{2 R}^{-}+\boldsymbol{\tau}\left(L_{R}\right)\left[\mathbf{R}_{R}^{11} \mathbf{p}_{2 R}^{+}+\mathbf{R}_{R}^{11} \boldsymbol{\tau}\left(L_{R}\right)\left(\mathbf{p}_{2 L}^{+}+\boldsymbol{\tau}\left(L_{L}\right) \mathbf{R}_{L}^{22} \mathbf{p}_{L}^{-}\right)\right]\right\} \\
& \mathbf{a}_{2 R}^{+}=\mathbf{p}_{2 L}^{+}+\boldsymbol{\tau}\left(L_{L}\right) \mathbf{R}_{L}^{22} \mathbf{p}_{2 L}^{-}+\boldsymbol{\tau}\left(L_{L}\right) \mathbf{R}_{L}^{22} \boldsymbol{\tau}\left(L_{L}\right) \mathbf{a}_{2 L}^{-}
\end{aligned}
$$

Therefore the total reaction charge is

$$
Q=\tilde{\mathbf{K}}^{\varphi \varphi} v L-\int_{0}^{L}\left[\tilde{\mathbf{K}}_{0}^{\varphi u} \mathbf{q}(z)-\jmath k \tilde{\mathbf{K}}_{1}^{\varphi u} \mathbf{q}(z)\right] \mathrm{d} z
$$

where $\mathbf{q}\left(z_{i}\right)=\boldsymbol{\Phi}^{+} \mathbf{a}_{2 R}^{+}+\boldsymbol{\Phi}^{-} \mathbf{a}_{2 L}^{-}$.

The integral in Eq. (35) can be evaluated numerically and current is recovered after differentiating the reaction charge with respect to time.

\section{EXPERIMENTS}

The framework proposed in this paper was validated with an experiment on a piezo-equipped beam with emulated anechoic terminations. A schematic diagram of the experimental set-up is shown in Fig. 7. The piezoelectric actuator $(0.076 \mathrm{~m} \times 0.0232 \mathrm{~m} \times 0.0022 \mathrm{~m}$ made of Noliac NCE40 material - see Table 1$)$ was driven by a signal from the generator module of a Polytec PSV300 controller amplified with a PCB 790 Series power amplifier.

Prior to bonding to the beam the impedance of the free-free actuator was measured. Corresponding numerical results were obtained from the wave model consisting of one $0.076 \mathrm{~m}$ long wave element with free-free boundaries. The impedance was extracted from the wave model according to the technique outlined in Section 4.2.

The manufacturer of the piezoelectric actuators states that the properties listed in the datasheet ${ }^{40}$ are for reference purposes only and the actual values depend on various conditions such as shape, finishing, electrode type etc. The quoted standard tolerances are $\pm 10 \%$ for electrical properties and $\pm 5 \%$ for mechanical properties. 


\begin{tabular}{llll}
\hline Property & Steel & Epoxy & NCE40 \\
\hline$E, \mathrm{GPa}$ & 163 & 15 & $\mathrm{n} / \mathrm{a}$ \\
$\nu$ & 0.3 & 0.4 & $\mathrm{n} / \mathrm{a}$ \\
$\rho, \mathrm{kg} \mathrm{m}^{-3}$ & 8000 & 1000 & 7850 \\
$\eta$ & 0.0001 & 0.0001 & 0.007 \\
$c_{x x}^{E}=c_{z z}^{E}, \mathrm{GPa}$ & $\mathrm{n} / \mathrm{a}$ & $\mathrm{n} / \mathrm{a}$ & 126.35 \\
$c_{x y}^{E}=c_{y z}^{E}, \mathrm{GPa}$ & $\mathrm{n} / \mathrm{a}$ & $\mathrm{n} / \mathrm{a}$ & 58.68 \\
$c_{y y}^{E}, \mathrm{GPa}$ & $\mathrm{n} / \mathrm{a}$ & $\mathrm{n} / \mathrm{a}$ & 99.88 \\
$c_{x z}^{E}, \mathrm{GPa}$ & $\mathrm{n} / \mathrm{a}$ & $\mathrm{n} / \mathrm{a}$ & 62.93 \\
$c_{x z}^{E}, \mathrm{GPa}$ & $\mathrm{n} / \mathrm{a}$ & $\mathrm{n} / \mathrm{a}$ & 31.71 \\
$c_{y z}^{E}=c_{x y}^{E}, \mathrm{GPa}^{E}$ & $\mathrm{n} / \mathrm{a}$ & $\mathrm{n} / \mathrm{a}$ & 36.77 \\
$\varepsilon_{x x}^{\epsilon}=\varepsilon_{z z}^{\epsilon}, \mathrm{F} \mathrm{m}^{-1}$ & $\mathrm{n} / \mathrm{a}$ & $\mathrm{n} / \mathrm{a}$ & $5.5 \mathrm{e}-09$ \\
$\varepsilon_{y y}^{\epsilon}, \mathrm{F} \mathrm{m}^{-1}$ & $\mathrm{n} / \mathrm{a}$ & $\mathrm{n} / \mathrm{a}$ & $5.196 \mathrm{e}-09$ \\
$e_{y x x}=e_{y z z}, \mathrm{~N} \mathrm{~V}^{-1} \mathrm{~m}^{-1}$ & $\mathrm{n} / \mathrm{a}$ & $\mathrm{n} / \mathrm{a}$ & -3.239 \\
$e_{z y z}=e_{x y x}, \mathrm{~N} \mathrm{~V}^{-1} \mathrm{~m}^{-1}$ & $\mathrm{n} / \mathrm{a}$ & $\mathrm{n} / \mathrm{a}$ & 13.075 \\
$e_{y y y}, \mathrm{~N} \mathrm{~V}^{-1} \mathrm{~m}^{-1}$ & $\mathrm{n} / \mathrm{a}$ & $\mathrm{n} / \mathrm{a}$ & 16.335 \\
\hline & & & \\
\hline
\end{tabular}

Table 1: Material constants used for experimental validation.
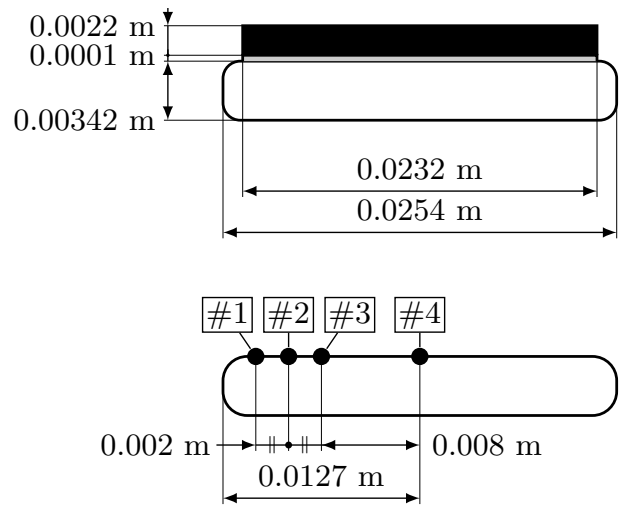

Figure 6: Cross-section of the beam with and without the PZT layer - dimensions and scan points locations.

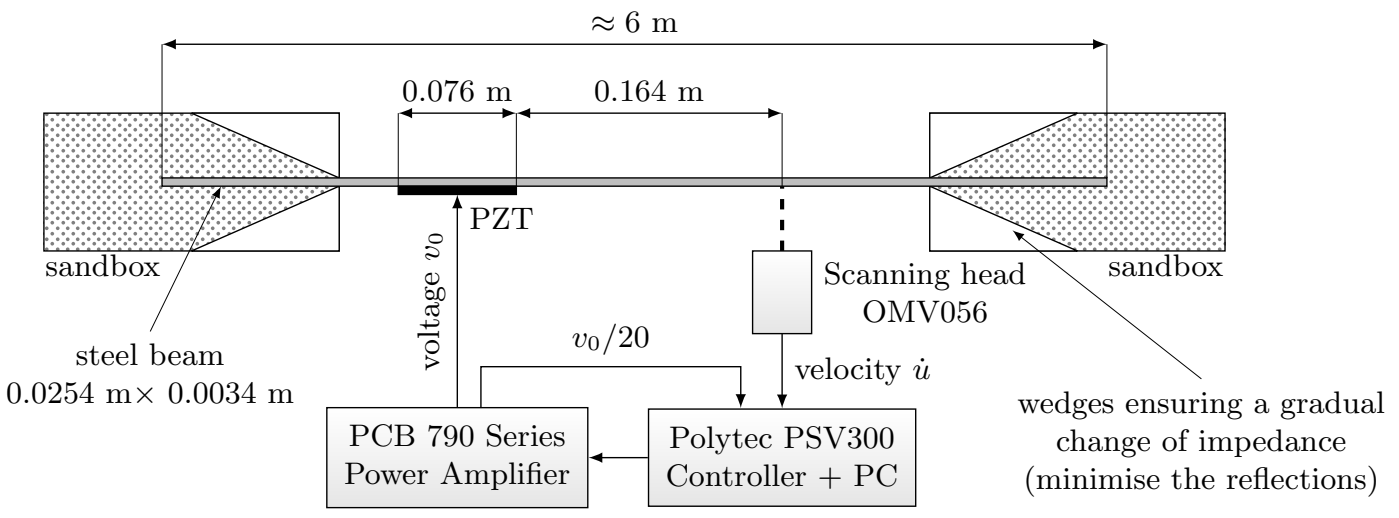

Figure 7: Experimental setup.

The properties of NCE40 from ${ }^{40}$ were updated based on the free-free actuator impedance measurement to provide the closest match (within stated tolerances) giving the values listed in Tab. 1.

The updated wave model prediction is compared with the measured data in Fig. 8. The electrical impedance contains imprints of all strain components, therefore can serve as a validity indicator for a 3D wave field. The response obtained from the wave model is slightly overdamped at high frequencies which is attributed to the effect of the constant loss factor used in the SAFE routine to represent damping. ${ }^{28}$

The actuator was attached to the beam using a thin (ca. $0.1 \mathrm{~mm}$ - inferred from thickness measurement) layer of silvered epoxy (CircuitWorks CW2400 - properties of the epoxy were not measured and typical values were adopted instead). Since the beam was a standard rolled mild steel profile, its cross-section was not ideally rectangular (see Fig 6). The SAFE-wave model represents the cross-section with a rectangle, therefore to account for that, the width in the model was amended and taken to be $0.024 \mathrm{~m}$. All relevant material properties are listed in Table 1.

During the test the response to the electrical excitation was measured at $0.164 \mathrm{~m}$ from the actuator and at a few locations along the width of the beam using a Polytec PSV300 laser vibrometer. The grid of scanner points was chosen to be close to the positions of the nodes in the wave model (see Fig. 6). The ends of the beam were placed in boxes filled with sand in such a way that the thickness of the sand cover was gradually increased to ensure a smooth change in the mechanical impedance of the boundary and minimise any reflections.

The corresponding wave model consisted of three wave elements (as described in Section 4): two singlelayer (steel) sections and one three-layer (piezo-bonding-steel) section. Appropriate continuity conditions at the 

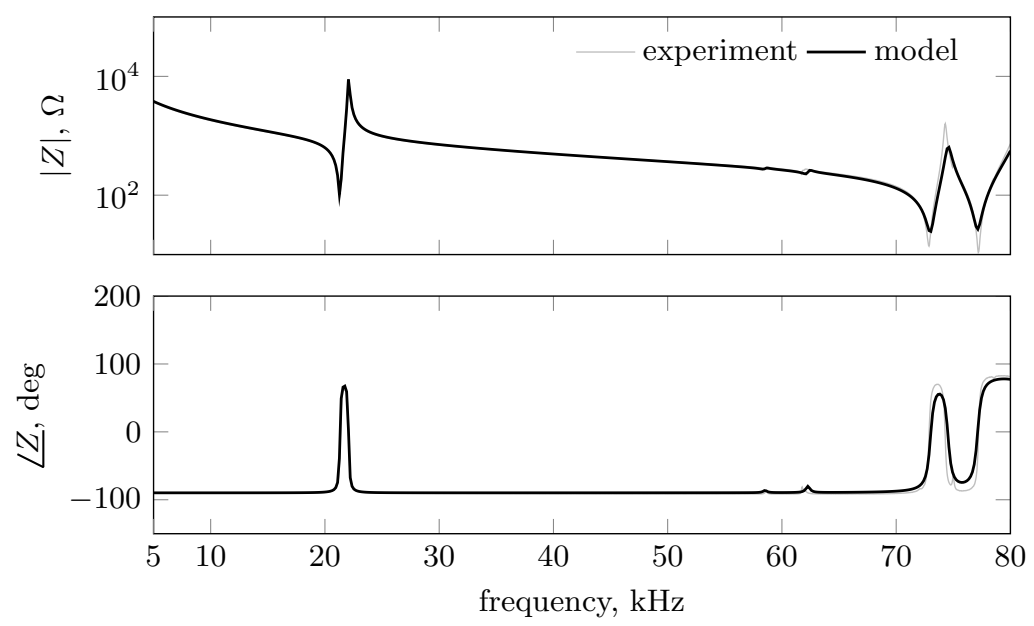

Figure 8: The electrical impedance of the free-free actuator prior to bonding.

junctions of the wave elements were specified. Each cross-section was modelled with six 8-node biquadratic quadrilateral elements across the width and one element across the thickness of each layer.

Recorded mobilities are compared with the wave model predictions in Figs. 9a-9d. The parts of the mobilities where simple bending is dominant $(<20 \mathrm{kHz})$ are not presented in Figs. 9b-9d, as they do not differ from Fig. 9a.

If the terminations were perfectly anechoic, the measured response would only show the waves passing through the position of the laser dot. In reality, the absorption is not perfect which imposes the fluctuations on the response. We observed that the wave absorption in the sandboxes largely depended on the amplitude of the excited waves indicating that the sandboxes behaved nonlinearly. Nevertheless, the mobilities presented in Figs. 9a-9d show predominantly the effect of the propagating waves.

At low frequencies the mobility at the center of the beam (Fig. 9a) follows the behaviour expected from the approximate theories with simple bending being dominant. The first sudden jump in magnitude at around 27 $\mathrm{kHz}$ corresponds to the cut-off frequency of the first transverse bending mode in the steel beam. At around 35 $\mathrm{kHz}$ the mobility reaches its maximum which is related to the cut-off frequency of the first transverse bending wave in the PZT-covered section. The response experiences a dip whenever the length of the actuator matches with a multiple of the predominantly excited wavelength. Figs. 9b-9d show the response measured at different locations across the width of the beam (as indicated).

In order to show the potential of the proposed approach, the experimental and numerical mobilities are supplemented with the results obtained from a model adopted from the literature. ${ }^{4}$ Predictions obtained with the Timoshenko-Mindlin-Hermann model ${ }^{4}$ agree well with the experiment up to ca. $5 \mathrm{kHz}$ but deviate at higher frequencies, since the model from the literature is developed under the assumptions of beam/rod theories. Our model enables gaining more physical insight into wave motion than the approaches originating from the pinforce assumption or various approximate theories. The conventional approaches are not able to represent full dynamics of the cross-section and to capture the higher-order waves (e.g. the one cutting of at ca. $35 \mathrm{kHz}$ ) which in structural waveguides often govern the response at ultrasonic frequencies.

The comparison for other locations along the width of the beam show a very good agreement between the wave model and the experiment. A slight drift in the phase at high frequencies can be partially attributed to the phase wrapping routines and difficulty in capturing the phase change at cut-off frequencies correctly. The fact that the phase slopes of both lines are almost identical is a better indication of the correspondence of the two results. 

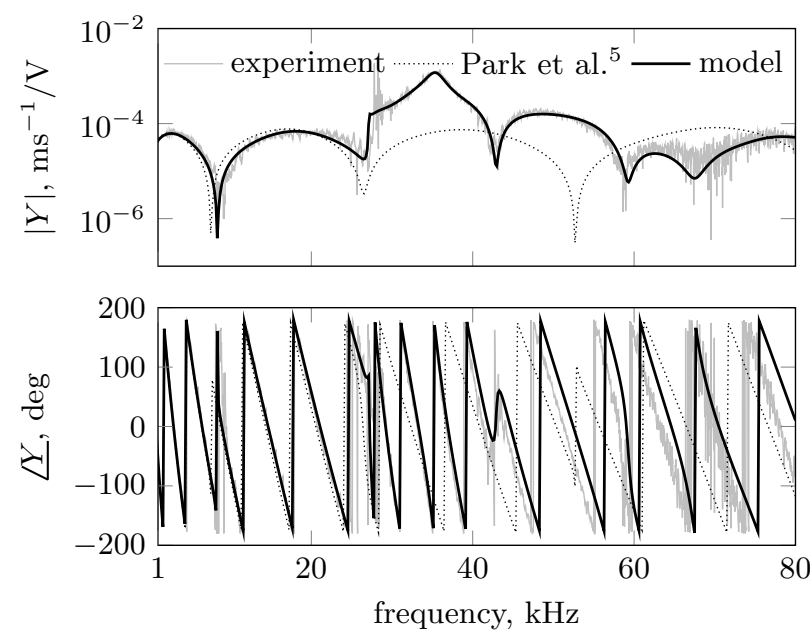

(a) scan point \#4
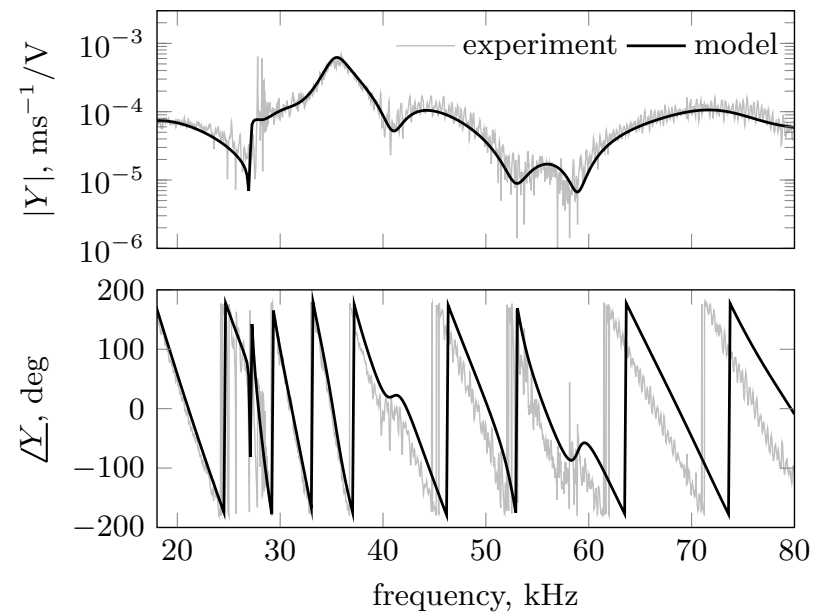

(c) scan point \#2
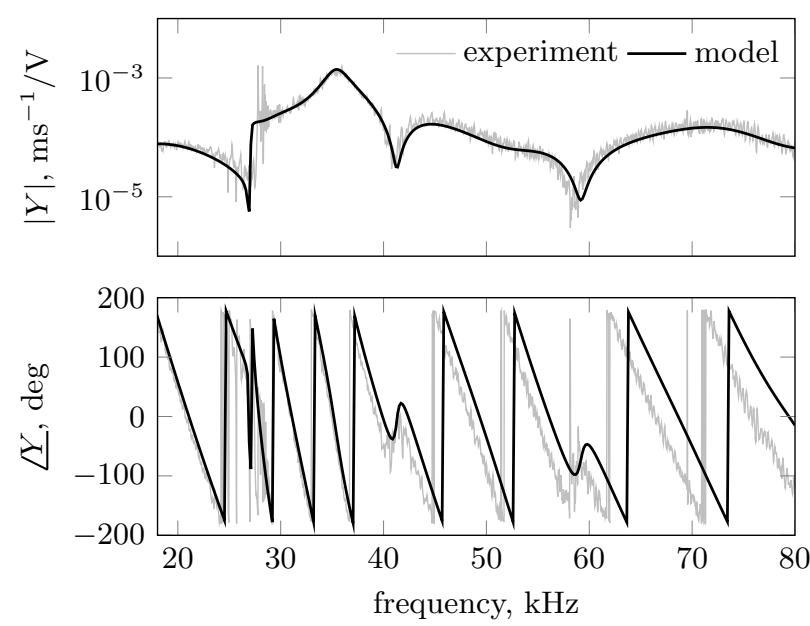

(b) scan point \#1
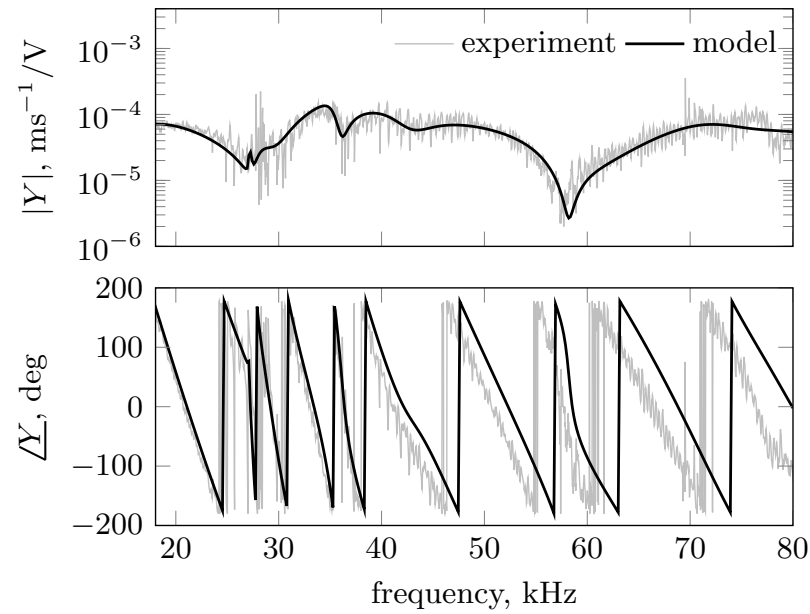

(d) scan point \#3

Figure 9: Magnitude and phase of the mobility with respect to the driving voltage measured at scan point marked in Fig. 6.

\section{CONCLUSIONS}

The methodology for modelling piezoelectric excitation in structural waveguides was developed and validated in this paper. The coupling of SAFE and the analytical wave approach enabled dropping the commonly used quasi-static and pin-force assumptions and including the actuator's dynamics in the wave model. Thanks to that the actuator is allowed to be thick (convergence of SAFE must be ensured though) and to operate at frequencies close to its resonance. The mathematical framework for calculation of the steady-state response to a distributed voltage excitation was also outlined.

The electromechanical wave model was validated with an experiment. Piezo-actuated structural waves in a beam-like waveguide with emulated anechoic terminations were captured using a laser vibrometer at different locations and compared with the numerical calculation. The comparison showed a very good agreement and proved the validity of the proposed approach. 


\section{ACKNOWLEDGMENTS}

This research is possible thanks to the University of Southampton Postgraduate Research Studentship Programme. Financial support of Structural Funds in the Operational Programme - Innovative Economy (IE OP) financed from the European Regional Development Fund - Project "Modern material technologies in aerospace industry", Nr POIG.01.01.02-00-015/08-00 is gratefully acknowledged.

\section{REFERENCES}

[1] Huang, G., Song, F., and Wang, X., "Quantitative modeling of coupled piezo-elastodynamic behavior of piezoelectric actuators bonded to an elastic medium for structural health monitoring: A review," Sensors 10, 3681-3702 (Apr. 2010).

[2] Moll, J., Golub, M. V., Glushkov, E., Glushkova, N., and Fritzen, C.-P., "Non-axisymmetric Lamb wave excitation by piezoelectric wafer active sensors," Sensors and Actuators A: Physical 174, 173-180 (Feb. 2012).

[3] Crawley, E. F. and De Luis, J., "Use of piezoelectric actuators as elements of intelligent structures," AIAA Journal 25, 1373-1385 (Oct. 1987).

[4] Park, H. W., Kim, E. J., Lim, K. L., and Sohn, H., "Spectral element formulation for dynamic analysis of a coupled piezoelectric wafer and beam system," Computers $\mathscr{6}$ Structures 88, 567-580 (May 2010).

[5] Park, I. and Lee, U., "Dynamic analysis of smart composite beams by using the frequency-domain spectral element method," Journal of Mechanical Science and Technology 26, 2511-2521 (Aug. 2012).

[6] Lee, U., Kim, D., and Park, I., "Dynamic modeling and analysis of the PZT-bonded composite timoshenko beams: Spectral element method," Journal of Sound and Vibration 332, 1585-1609 (Mar. 2013).

[7] Giurgiutiu, V., [Structural health monitoring: with piezoelectric wafer active sensors], Access Online via Elsevier (2007).

[8] Raghavan, A. and Cesnik, C. E., "Analytical models for Lamb waves based structural health monitoring," in [Proceedings of the 23rd international modal analysis conference (IMAX XXIII), Paper], (2005).

[9] Giurgiutiu, V., "Tuned Lamb wave excitation and detection with piezoelectric wafer active sensors for structural health monitoring," Journal of Intelligent Material Systems and Structures 16, 291-305 (Apr. 2005).

[10] Scalea, F. L. d. and Salamone, S., "Temperature effects in ultrasonic Lamb wave structural health monitoring systems," The Journal of the Acoustical Society of America 124, 161-174 (July 2008).

[11] Yu, L., Bottai-Santoni, G., and Giurgiutiu, V., "Shear lag solution for tuning ultrasonic piezoelectric wafer active sensors with applications to Lamb wave array imaging," International Journal of Engineering Science 48, 848-861 (Oct. 2010).

[12] Santoni-Bottai, G. and Giurgiutiu, V., "Exact shear-lag solution for guided waves tuning with piezoelectricwafer active sensors," AIAA Journal 50, 2285-2294 (Nov. 2012).

[13] Glushkov, E., Glushkova, N., Kvasha, O., and Seemann, W., "Integral equation based modeling of the interaction between piezoelectric patch actuators and an elastic substrate," Smart Materials and Structures 16, 650-664 (June 2007).

[14] Kim, Y., Ha, S., and Chang, F.-K., "Time-domain spectral element method for built-in piezoelectricactuator-induced Lamb wave propagation analysis," AIAA Journal 46, 591-600 (Mar. 2008).

[15] Ostachowicz, W., Kudela, P., Krawczuk, M., and Zak, A., [Guided Waves in Structures for SHM: The Time-Domain Spectral Element Method], John Wiley \& Sons, Ltd (2012).

[16] Moulin, E., Assaad, J., Delebarre, C., and Osmont, D., "Modeling of Lamb waves generated by integrated transducers in composite plates using a coupled finite elementnormal modes expansion method," The Journal of the Acoustical Society of America 107, 87-94 (Jan. 2000).

[17] Pako, P., Bielak, T., Spencer, A. B., Staszewski, W. J., Uhl, T., and Worden, K., "Lamb wave propagation modelling and simulation using parallel processing architecture and graphical cards," Smart Materials and Structures 21, 075001 (July 2012).

[18] Auld, B., [Acoustic Fields in Elastic Solids, Vol. I, II], Wiley-Interscience (1973). 
[19] Cremer, L. and Heckl, M., [Structure-borne sound; structural vibrations and sound radiation at audio frequencies], Springer-Verlag (1973).

[20] Mace, B. R., "Wave reflection and transmission in beams," Journal of Sound and Vibration 97, 237-246 (Nov. 1984).

[21] Waki, Y., On the application of finite element analysis to wave motion in one-dimensional waveguides, $\mathrm{PhD}$ thesis, University of Southampton (2007).

[22] Harland, N., Mace, B., and Jones, R., "Wave propagation, reflection and transmission in tunable fluid-filled beams," Journal of Sound and Vibration 241, 735-754 (Apr. 2001).

[23] Kalkowski, M., Piezo-actuated structural waves for delaminating surface accretions, PhD thesis, University of Southampton. Faculty of Engineering and the Environment. Institute of Sound and Vibration Research (January 2015).

[24] Lagasse, P. E., "Higher-order finite-element analysis of topographic guides supporting elastic surface waves," The Journal of the Acoustical Society of America 53(4), 1116-1122 (1973).

[25] Aalami, B., "Waves in prismatic guides of arbitrary cross section," Journal of Applied Mechanics 40, 10671072 (Dec. 1973).

[26] Hayashi, T., Song, W.-J., and Rose, J. L., "Guided wave dispersion curves for a bar with an arbitrary cross-section, a rod and rail example," Ultrasonics 41, 175-183 (May 2003).

[27] Damljanovic, V. and Weaver, R. L., "Forced response of a cylindrical waveguide with simulation of the wavenumber extraction problem," The Journal of the Acoustical Society of America 115(4), 1582 (2004).

[28] Bartoli, I., Marzani, A., Lanza di Scalea, F., and Viola, E., "Modeling wave propagation in damped waveguides of arbitrary cross-section," Journal of Sound and Vibration 295, 685-707 (Aug. 2006).

[29] Gao, H., Ultrasonic guided wave mechanics for composite material structural health monitoring, $\mathrm{PhD}$ thesis, Pennsylvania State University (2007).

[30] Le Crom, B. and Castaings, M., "Shear horizontal guided wave modes to infer the shear stiffness of adhesive bond layers," The Journal of the Acoustical Society of America 127(4), 2220 (2010).

[31] Gaudenzi, P., [Smart Structures: Physical Behaviour, Mathematical Modelling and Applications], John Wiley \& Sons (Oct. 2009).

[32] Gaudenzi, P. and Bathe, K.-J., "An iterative finite element procedure for the analysis of piezoelectric continua," Journal of Intelligent Material Systems and Structures 6, 266-273 (Mar. 1995).

[33] Allik, H. and Hughes, T. J. R., "Finite element method for piezoelectric vibration," International Journal for Numerical Methods in Engineering 2(2), 151-157 (1970).

[34] Kocbach, J., Finite Element Modeling of Ultrasonic Piezoeelctric Transducers, PhD, University of Bergen (2000).

[35] Piefort, V., Finite element modelling of piezoelectric active structures, $\mathrm{PhD}$ thesis, Universit Libre de Bruxelles (2001).

[36] Boucher, D., Lagier, M., and Maerfeld, C., "Computation of the vibrational modes for piezoelectric array transducers using a mixed finite element-perturbation method," Sonics and Ultrasonics, IEEE Transactions on 28, 318-329 (Sept. 1981).

[37] Tisseur, F. and Meerbergen, K., "The quadratic eigenvalue problem," SIAM Review 43, 235-286 (Jan. 2001).

[38] Renno, J. M. and Mace, B. R., "On the forced response of waveguides using the wave and finite element method," Journal of Sound and Vibration 329, 5474-5488 (Dec. 2010).

[39] Morse, P. M., [Theoretical Acoustics], Princeton University Press (1986).

[40] Noliac Group, "Piezo materials datasheet." 